\title{
松濑退化草地芦苇不同叶位叶片营养元素代谢特征
}

\author{
郭 瑞 ${ }^{1,2 *}$ 周 际 ${ }^{3}$ 刘 琪 ${ }^{1,2}$ 顾峰雪 1,2
}

${ }^{1}$ 中国农业科学院农业环境与可持续发展研究所, 北京 $100081 ;{ }^{2}$ 农业部旱作节水农业重点实验室, 北京 $100081 ;{ }^{3}$ 国土资源部土地整治中心, 北京 100034

摘 要 对松嫩草地不同退化程度样地的芦苇(Phragmites australis)各叶位叶片的生长及营养元素代谢特征进行分析, 以探

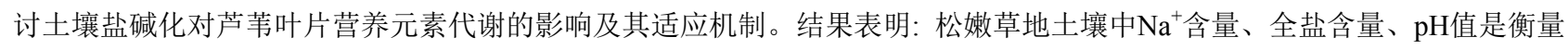
土壤盐碱化程度的主要决定因子, 从典型草地到重度退化草地, 土壤盐碱化程度逐级加剧。芦苇具有一定程度的耐盐碱性, 植株高度和地上部分生物量随土壤盐分增加而降低。检测出 10 种营养元素: $\mathrm{K} 、 \mathrm{Na} 、 \mathrm{Ca} 、 \mathrm{Mg} 、 \mathrm{Fe} 、 \mathrm{Cu} 、 \mathrm{Zn} 、 \mathrm{Mn} 、 \mathrm{P} 、 \mathrm{~B}$, 主 成分分析结果显示全部样本均处于 $95 \%$ 的置信区间内。方差分析结果表明, 芦苇不同叶位叶片对营养元素的富集能力有所差 异。 $\mathrm{K} 、 \mathrm{P}$ 含量随叶位降低而减少; 而 $\mathrm{Na} 、 \mathrm{Ca} 、 \mathrm{Mg}$ 呈现相反分布规律。 $\mathrm{Fe} 、 \mathrm{Cu}$ 集中分布在功能叶和老叶中; $\mathrm{Mn}$ 只大量聚集在 老叶中; 而 $\mathrm{Zn}$ 集中分布在幼叶中。表明土壤盐碱化对老叶营养元素的影响大于幼叶, $\mathrm{Na}$ 在老叶中的大量积累保护了幼叶免于 或者减轻离子的毒害。功能叶和老叶中 $\mathrm{Ca} 、 \mathrm{Mg} 、 \mathrm{Fe} 、 \mathrm{Cu}$ 的积累有利于保障芦苇正常的光合作用。盐碱胁迫下幼叶仍维持较 高 $\mathrm{K} 、 \mathrm{P}$ 含量, 这不仅为幼叶的生长提供所需营养, 同时提高了其抗逆性, 这可能是芦苇的生理响应策略。

关键词 松嫩草地; 芦苇; 营养元素; 叶位; 退化草地

郭瑞, 周际, 刘琪, 顾峰雪 (2018). 松嫩退化草地芦苇不同叶位叶片营养元素代谢特征. 植物生态学报, 42, 734-740. DOI: 10.17521/cjpe.2018.0061

\section{Characterization of nutrient elements at different leaf positions in Phragmites australis in Songnen degraded grassland}

GUO Rui ${ }^{1,2^{*}}$, ZHOU Ji ${ }^{3}$, LIU Qi ${ }^{1,2}$, and GU Feng-Xue ${ }^{1,2}$

${ }^{1}$ Institute of Environment and Sustainable Development in Agriculture, Chinese Academy of Agricultural Sciences, Beijing 100081, China; ${ }^{2}$ Key Laboratory of Dryland Agriculture, Ministry of Agriculture, Beijing 100081, China; and ${ }^{3}$ Land Consolidation and Rehabilitation Centre, the Ministry of Land and Resources, Beijing 100034, China

\section{Abstract}

Aims This study was performed to compare the growth and characteristics of nutrient elements at different leaf positions of reed (Phragmites australis) under salinity stress. The adaptive mechanisms by which reeds at a Songnen grassland tolerate salinity stress were determined.

Methods Samples were collected from normal grassland, moderate degraded grassland and severe degraded grassland, respectively. Plant height, shoot dry mass, and nutrient elements at different leaf positions of reeds were measured.

Important findings The $\mathrm{Na}^{+}$content, total salt content and $\mathrm{pH}$ value of soil are important factors that determine soil salinity in the Songnen grassland. The degree of soil salinity was aggravated stepwise from the single dominant reed communities to the reed coexisted with Suaeda salsa communities. Height and shoot dry mass decreased with increased salinity. The profiles of 10 key nutrient elements were measured, namely, $\mathrm{K}, \mathrm{Na}, \mathrm{Ca}, \mathrm{Mg}$, $\mathrm{Fe}, \mathrm{Cu}, \mathrm{Zn}, \mathrm{Mn}, \mathrm{P}$, and B. Results from the principal component analysis indicated that all samples were distributed within the $95 \%$ confidence interval. The results showed that $\mathrm{K}$ and $\mathrm{P}$ contents decreased with lowered leaf positions; while $\mathrm{Na}, \mathrm{Ca}$, and $\mathrm{Mg}$ showed the opposite distribution trends. $\mathrm{Fe}$ and $\mathrm{Cu}$ contents were less in young leaves than in the other leaves, $\mathrm{Mn}$ accumulated in the old leaves. However, a large amount of $\mathrm{Zn}$ accumulated in the young leaves. No obvious change in B was observed. Thus, salinity stress produced a small effect on the distribution of nutrients in young leaves, but strongly affected the older leaves. Under salinity stress, old leaves accumulated more $\mathrm{Na}^{+}$to protect the young leaves from ion harm. High accumulation of $\mathrm{Ca}, \mathrm{Mg}, \mathrm{Fe}$, and $\mathrm{Cu}$ in mature and old leaves was more beneficial to maintain and protect the function of photosynthesis. The increased $\mathrm{K}$ and $\mathrm{P}$ contents in the young leaves could provide the necessary nutrition and improve the salinity resistance of

收稿日期Received: 2018-03-22 接受日期Accepted: 2018-05-12

基金项目: 国家自然科学基金(31570328)。Supported by the National Natural Science Foundation of China (31570328).

* E-mail: guorui01@caas.cn 
reed. This process contributes to the physiological response strategy of reed under salinity stress.

Key words Songnen grassland; Phragmites australis; nutrient elements; leaves positions; degraded grassland

Guo R, Zhou J, Liu Q, Gu FX (2018). Characterization of nutrient elements at different leaf positions in Phragmites australis in Songnen degraded grassland. Chinese Journal of Plant Ecology, 42, 734-740. DOI: 10.17521/cjpe.2018.0061

芦苇(Phragmites australis)是一种多年生禾本科 植物, 广泛分布在世界各地, 从江河湖泊、低洼沼泽 地到荒漠、盐碱地均可生长, 它有较强的耐受性、 适应性和生存能力, 并进化出不同生态型而成为世 界性物种(杨允菲和郎惠卿, 1998)。芦苇具有较强的 生态功能，水生芦苇可吸附重金属和分解有机污染 物从而净化水质, 旱生芦苇能够固沙和改善土壤环 境; 同时芦苇还拥有很高的经济价值, 它产量高, 适口性好, 是优良饲料, 另外, 其纤维含量高, 是不 可缺少的造纸原料(庄瑶等, 2010; Kiviat, 2013)。

松嫩平原地处温带, 位于东北平原中心, 三面 分别被长白山、大兴安岭和小兴安岭包围(海霞等, 2004)。松嫩平原形成于全新世晚期, 由松花江和嫩 江冲积形成, 大量可溶性盐沉积和汇集形成盐碱化 草地(李秀军等, 2002)。松嫩平原土壤类型多样, 苏 打盐渍化情况严重, 表层土壤含盐量大于 $3 \mathrm{~g} \cdot \mathrm{kg}^{-1}$, 具有东重西轻的空间分布特点, 是我国面积最大的 盐碱土集中地, 也是世界三大苏打盐碱土地之一 (张士功等, 2000)。苏打盐碱土中盐分主要由 $\mathrm{Na}_{2} \mathrm{CO}_{3}$ 和 $\mathrm{NaHCO}_{3}$ 构成, 同时含有少量的硫酸盐和氯化物, 苏打盐碱土对植物来说兼有中性盐和碱性盐双重胁 迫特点(郑慧莹和李建东, 1995; 赵兰坡等, 2000)。约 80 万年前芦苇出现在松嫩平原, 它广泛分布在湿 地、沙地及盐碱地, 能够维护生物种群多样性、巩 固和改善土壤、调节局部小气候, 松嫩平原上的芦 苇草地也可作为牧场或割草地, 因此芦苇在维持其 生态系统稳定性和平衡性方面发挥着重要作用, 同 时也影响当地农牧业的发展(杨允菲和郎惠卿, 1998; 邱天, 2014)。

目前, 针对芦苇的研究越来越受到重视, 在形 态结构、生理生态、分子遗传、栽培管理等方面已 有大量相关报道, 但缺少针对自然盐碱环境下芦苇 新老器官营养元素代谢特征的研究分析(祥明等, 2000; Asaeda et al., 2002; 杨帆等, 2006; 庄瑶等, 2010)。鉴于此, 本研究从生长及营养元素分布角度, 分析和比较松嫩平原不同退化草地上芦苇幼叶、功 能叶、老叶中营养元素代谢调控特征及其适应机制。

\section{1 材料和方法}

\section{1 研究地区自然概况}

研究地点位于松嫩平原中部, 地势平坦开阔, 海拔高度约为 $145 \mathrm{~m}$ 。该地区以淡黑钙土为主, 兼有 风沙土、草甸土、盐碱土。该地区属于半干旱温带 季风气候, 年平均气温 $4.9{ }^{\circ} \mathrm{C}$, 年降水量约为 $400 \mathrm{~mm}$, 但平均年蒸发量约为 $1600 \mathrm{~mm}$, 远大于降 水量(赵兰坡等, 2000)。

\section{2 样地类型}

样地 1 : 典型草地(芦苇为优势种); 样地 2 : 中等 退化草地(芦苇与羊草(Leymus chinensis)伴生); 样 地3: 重度退化草地(芦苇与碱蓬(Suaeda glauca)伴 生)。每个样地内含 5 个 $1 \mathrm{~m} \times 1 \mathrm{~m}$ 样方, 每个样方相 距间隔 $<100 \mathrm{~cm}$ ，以保证 4 个样方具有相同的土壤、 气候特点。每一个样方设定为一个重复。

\section{3 样品取样}

\subsection{1 土壤取样}

在每个样方中取 $0-10 、 10-20 、 20-30 、 30-40 \mathrm{~cm}$ 的土壤样品, 并将其混匀、剔除杂质、风干磨细过 $0.85 \mathrm{~mm}$ 孔径土壤篮，按水土质量比 $5: 1$ 配置土壤溶 液, 均匀振荡后静置, 取上清液, 待测。

\subsection{2 芦苇取样}

在每个样方中随机选取1株芦苇, 测量株高后 分别取幼叶、功能叶、老叶, 取样后立刻放入液氮 中保存, 回到实验室后 $105{ }^{\circ} \mathrm{C}$ 杀青 $10 \mathrm{~min}$, 随后真 空低温干燥至恒质量, 称量待测。

\section{4 指标测定}

\subsection{1 土壤盐碱指标测定}

利用PHS-3C 型数字 $\mathrm{pH}$ 计 (虹益公司, 上海)和 DDG-2080-S电导仪(博取公司, 上海)测定土壤样品 上清液的 $\mathrm{pH}$ 值和电导率。利用原子吸收分光光度计 (TAS-990, 普析通用公司, 北京)测定土壤样品上清 液中 $\mathrm{K}^{+} 、 \mathrm{Na}^{+} 、 \mathrm{Ca}^{2+} 、 \mathrm{Mg}^{2+}$ 含量。应用双指示剂(酚 酞和溴酚蓝)中和滴定法测定 $\mathrm{CO}_{3}^{2-}$ 和 $\mathrm{HCO}_{3}^{-}$含量, 利 用离子色谱法(DX-300离子色谱仪, Thermo Fisher Scientific, Waltham, USA; AS4A-SC色谱柱, Dionex, Sunnyvale, $\mathrm{USA})$ ) 测定 $\mathrm{Cl}^{-}$和 $\mathrm{SO}_{4}^{2-}$ 含量。 


\subsection{2 营养元素测定}

称取叶片干样 $50 \mathrm{mg}$, 用硝酸在 $95{ }^{\circ} \mathrm{C}$ 条件下硝 化, 硝化后定容到 $10 \mathrm{~mL}$ 。利用Prodigy型电感耦合 等离子体原子发射光谱仪(ICP-AES, LEEMAN, New Hampshire, USA)测定K、Na、Ca、Mg、Fe、

$\mathrm{Cu} 、 \mathrm{Zn} 、 \mathrm{Mn} 、 \mathrm{P} 、 \mathrm{~B}$ 含量。

\section{5 数据分析}

利用SPSS 17.0对生长参数和营养元素指标进 行数据统计和分析, 每个实验结果为 5 次重复的平 均值(平均值土标准误差), $p<0.05$ 显著性差异。利用 MetaboAnalyst对营养元素进行数据分析, 主成分分 析(PCA)使用均值中心化, 结果用得分表示(Xia \& Wishart, 2011)。

\section{2 结果}

\section{1 土壤盐碱特征}

不同退化程度草地土壤的盐碱指标可以反映其 土壤特点。典型草地、中度退化草地、重度退化草 地土壤总盐分含量、 $\mathrm{pH}$ 值、电导率依次升高, 相互 之间呈现显著性差异(表 $1, p<0.05$ )。在 8 种离子中, 随土壤退化程度加深, $\mathrm{K}^{+} 、 \mathrm{Na}^{+} 、 \mathrm{Mg}^{2+} 、 \mathrm{CO}_{3}^{2-} 、 \mathrm{HCO}_{3}^{-}$、 $\mathrm{Cl}^{-} 、 \mathrm{SO}_{4}^{2-}$ 含量增加, 其中 $\mathrm{Na}^{+}$变化最为明显; 但 $\mathrm{Ca}^{2+}$ 含量降低(表1)。

表1 不同退化程度松嫩草地土壤盐碱特征(平均值土标准误差, $n=5$ )

Table 1 Soil salinity characteristics in the different degrees of degraded Songnen grasslands (mean $\pm S E, n=5$ )

\begin{tabular}{|c|c|c|c|}
\hline \multirow{2}{*}{$\begin{array}{l}\text { 土壤盐碱特征 } \\
\text { 和养分 } \\
\text { Soil salinity charac- } \\
\text { teristics and nutrient } \\
\text { contents }\end{array}$} & \multicolumn{3}{|c|}{$\begin{array}{c}\text { 不同退化程度松嫩草地 } \\
\text { The different degrees of retrogressive grassland }\end{array}$} \\
\hline & $\begin{array}{l}\text { 正常草地 } \\
\text { Normal } \\
\text { grassland }\end{array}$ & $\begin{array}{c}\text { 中度退化草地 } \\
\text { Moderate degra- } \\
\text { ded grassland }\end{array}$ & $\begin{array}{c}\text { 重度退化草地 } \\
\text { Severe degraded } \\
\text { grassland }\end{array}$ \\
\hline $\mathrm{K}^{+}\left(\mu \mathrm{mol} \cdot \mathrm{g}^{-1}\right)$ & $0.41 \pm 0.06^{\mathrm{c}}$ & $1.87 \pm 0.19^{b}$ & $2.73 \pm 0.24^{\mathrm{a}}$ \\
\hline $\mathrm{Na}^{+}\left(\mu \mathrm{mol} \cdot \mathrm{g}^{-1}\right)$ & $6.58 \pm 0.55^{\mathrm{c}}$ & $25.22 \pm 1.98^{\mathrm{b}}$ & $42.64 \pm 6.87^{\mathrm{a}}$ \\
\hline $\mathrm{Ca}^{2+}\left(\mu \mathrm{mol} \cdot \mathrm{g}^{-1}\right)$ & $1.41 \pm 0.10^{\mathrm{a}}$ & $1.01 \pm 0.09^{\mathrm{b}}$ & $0.78 \pm 0.02^{\mathrm{b}}$ \\
\hline $\mathrm{Mg}^{2+}\left(\mu \mathrm{mol} \cdot \mathrm{g}^{-1}\right)$ & $0.42 \pm 0.03^{\mathrm{c}}$ & $1.23 \pm 0.46^{\mathrm{b}}$ & $5.91 \pm 0.68^{\mathrm{a}}$ \\
\hline $\mathrm{CO}_{3}^{2-}\left(\mu \mathrm{mol} \cdot \mathrm{g}^{-1}\right)$ & $2.77 \pm 0.40^{\mathrm{c}}$ & $10.02 \pm 1.55^{b}$ & $18.35 \pm 2.76^{\mathrm{a}}$ \\
\hline $\mathrm{HCO}_{3}^{-}\left(\mu \mathrm{mol} \cdot \mathrm{g}^{-1}\right)$ & $9.34 \pm 0.76^{\mathrm{b}}$ & $21.11 \pm 1.98^{\mathrm{a}}$ & $23.24 \pm 3.65^{\mathrm{a}}$ \\
\hline $\mathrm{Cl}^{-}\left(\mu \mathrm{mol} \cdot \mathrm{g}^{-1}\right)$ & $1.66 \pm 0.23^{\mathrm{c}}$ & $5.87 \pm 0.55^{\mathrm{b}}$ & $16.06 \pm 4.01^{\mathrm{a}}$ \\
\hline $\mathrm{SO}_{4}^{2-}\left(\mu \mathrm{mol} \cdot \mathrm{g}^{-1}\right)$ & $0.53 \pm 0.01^{\mathrm{c}}$ & $2.21 \pm 0.54^{\mathrm{b}}$ & $4.36 \pm 0.47^{\mathrm{a}}$ \\
\hline TSC $\left(\mu \mathrm{mol} \cdot \mathrm{g}^{-1}\right)$ & $23.12 \pm 1.88^{\mathrm{c}}$ & $68.54 \pm 7.02^{b}$ & $114.07 \pm 16.32^{\mathrm{a}}$ \\
\hline $\mathrm{pH}$ 值 $\mathrm{pH}$ value & $8.72 \pm 0.37^{\mathrm{c}}$ & $9.55 \pm 0.83^{b}$ & $10.18 \pm 0.72^{\mathrm{a}}$ \\
\hline$E C\left(\mu \mathrm{s} \cdot \mathrm{cm}^{-1}\right)$ & $128.97 \pm 3.44^{\mathrm{c}}$ & $450.54 \pm 11.34^{\mathrm{b}}$ & $698.86 \pm 22.70^{\mathrm{a}}$ \\
\hline
\end{tabular}

$E C$, 电导率; TSC, 总盐分含量。不同小写字母表示不同退化草地间差异 显著 $(p<0.05)$ 。

$E C$, electrical conductivity; TSC, total salinity concentration. Different lowercase letters indicate significant differences among the different degrees of retrogressive soil $(p<0.05)$.

\section{2 芦苇生长特征}

盐碱胁迫下芦苇株高及地上部分干质量随草地 退化程度增加而下降，从正常草地到中等退化草地 下降幅度尤其明显(图 $1, p<0.05)$ 。

\section{3 营养元素变化轨迹}

PCA结果显示，正常草地、中等退化草地和严 重退化草地上的芦苇不同叶位叶片营养元素之间的 差异显著, 上部新叶、中部功能叶、下部老叶两个 主成分合计得分分别为 $84.6 \% 、 85.4 \% 、 90.3 \%$, 这表 明本研究试验设计和ICP-AES分析方法可以有效地 区分芦苇叶片在盐碱胁迫下的元素代谢特点(图2)。

\section{4 营养元素变化特征}

表2结果显示, 尽管生境不同, 但营养元素在芦 苇幼叶、功能叶和老叶中的分布规律相似。芦苇幼 叶中 $\mathrm{K}$ 含量远高于功能叶和老叶, 而功能叶和老叶 之间并无显著性差异; 但 $\mathrm{Na}$ 呈现相反分布趋势, 幼 叶中 $\mathrm{Na}$ 含量远低于其他叶片(表2)。Ca、 $\mathrm{Mg}$ 含量变
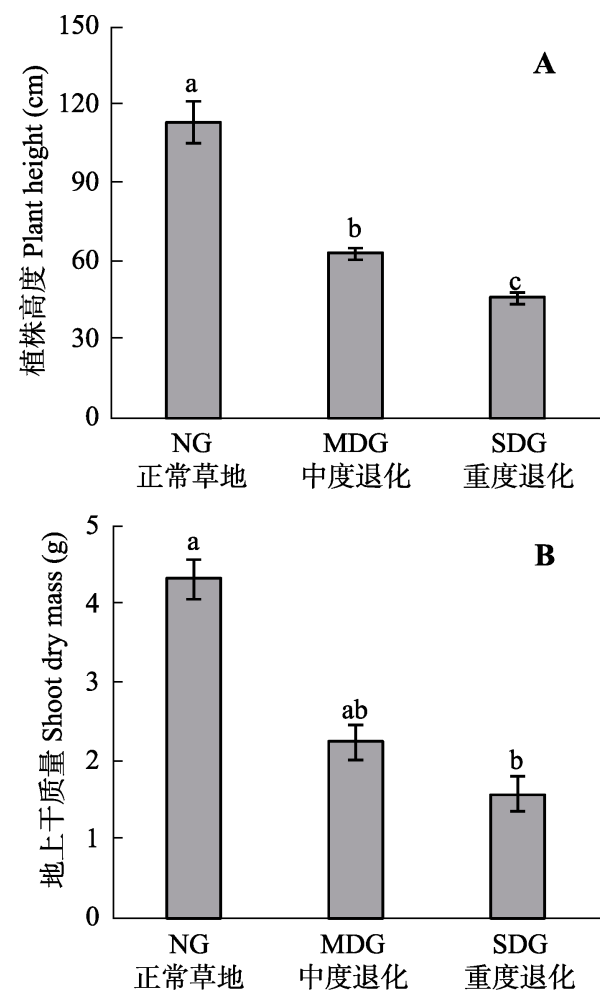

图1 不同退化程度草地芦苇植株高度和地上干质量的变化 趋势(平均值 \pm 标准误差, $n=5$ )。不同小写字母表示不同处理 间差异显著 $(p<0.05)$ 。 NG, 正常草地; MDG, 中度退化草地; SDG, 重度退化草地。

Fig. 1 Changes of plant height and shoot dry mass of Phragmites communis in different degrees of degraded grasslands (mean $\pm S E, n=5$ ). Different lowercase letters indicate significant differences among the different degrees of degraded grasslands $(p<0.05)$. NG, normal grassland; MDG, moderate degraded grassland; SDG, severe degraded grassland. 

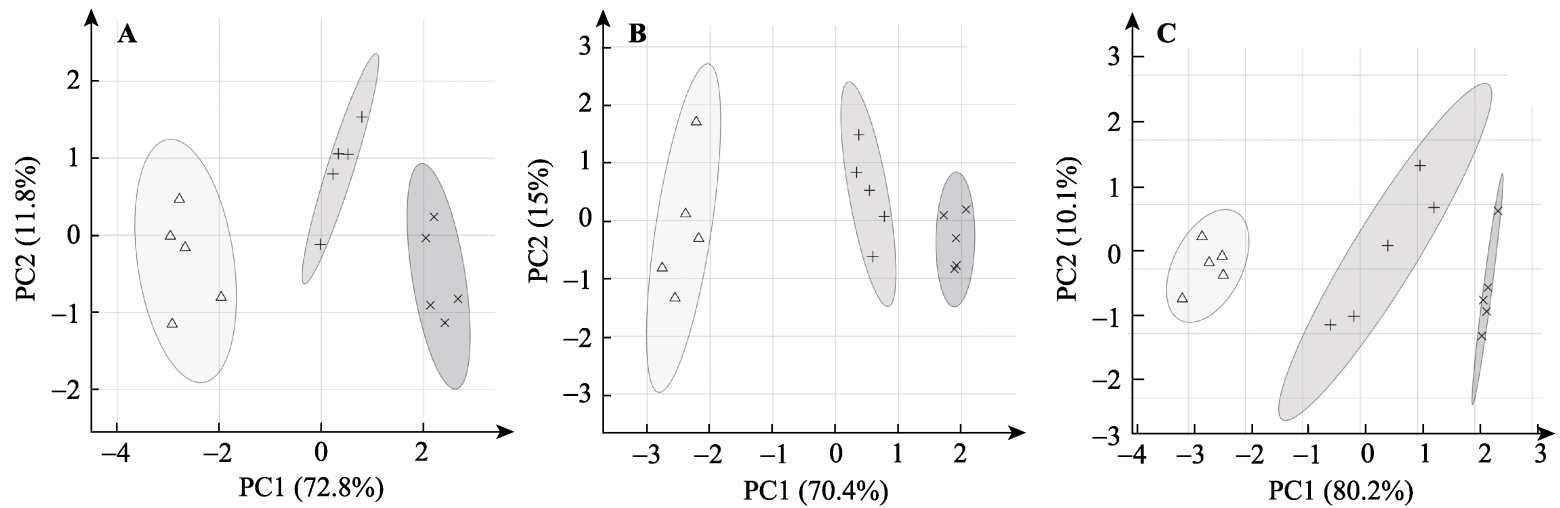

$\Delta$ 老叶 Old leaf + 功能叶 Mature leaf $\times$ 幼叶 Yong leaf

图2 主成分分析(PCA)得分图显示芦苇上部幼叶、中部功能叶和下部老叶在不同生境下的营养元素变化轨迹。 $\mathrm{A}$, 正常草地。 B, 中等退化草地。C, 严重退化草地。

Fig. 2 Principal component analysis (PCA) score plots showing the nutrition elements trajectory of young leaves, mature leaves and old leaves in different habitats. A, Normal grassland. B, Moderate degraded grassland. C, Severe degraded grassland.

表2 不同退化程度松嫩草地上芦苇各叶位叶片中营养元素含量 $\left(\mu \mathrm{mol} \cdot \mathrm{g}^{-1}\right.$ ) (平均值土标准误差, $n=5$ )

Table 2 The contents of nutrient elements in different leaf positions of Phragmites communis in the different degrees of degraded Songnen grasslands $\left(\mu \mathrm{mol} \cdot \mathrm{g}^{-1}\right)($ mean $\pm S E, n=5)$

\begin{tabular}{|c|c|c|c|c|c|c|c|c|c|}
\hline \multirow{3}{*}{$\begin{array}{l}\text { 营养 } \\
\text { 元素 } \\
\text { Nutrients } \\
\text { elements }\end{array}$} & \multicolumn{9}{|c|}{ 同退化程度松嫩草地不同叶位叶片 The different degrees of degraded grassland/The different leaf positions } \\
\hline & \multicolumn{3}{|c|}{ 正常草地 Normal grassland } & \multicolumn{3}{|c|}{ 中等退化草地 Moderate degraded grassland } & \multicolumn{3}{|c|}{ 严重退化草地 Severe degraded grassland } \\
\hline & $\begin{array}{c}\text { 幼叶 } \\
\text { Young leaf }\end{array}$ & $\begin{array}{c}\text { 功能叶 } \\
\text { Mature leaf }\end{array}$ & $\begin{array}{c}\text { 老叶 } \\
\text { Old leaf }\end{array}$ & $\begin{array}{c}\text { 幼叶 } \\
\text { Young leaf }\end{array}$ & $\begin{array}{c}\text { 功能叶 } \\
\text { Mature leaf }\end{array}$ & $\begin{array}{c}\text { 老叶 } \\
\text { Old leaf }\end{array}$ & $\begin{array}{c}\text { 幼叶 } \\
\text { Young leaf }\end{array}$ & $\begin{array}{c}\text { 功能叶 } \\
\text { Mature leaf }\end{array}$ & $\begin{array}{c}\text { 老叶 } \\
\text { Old leaf }\end{array}$ \\
\hline 钾 K & $0.54 \pm 21.69^{\mathrm{a}}$ & 301.5 & 27 & $38 \pm 11.5$ & $.71 \pm 8.10^{\mathrm{b}}$ & $77 \pm 39$. & $.01 \pm$ & $1.43=$ & - \\
\hline 钠 $\mathrm{Na}$ & $39 \pm 2.70^{\mathrm{c}}$ & $31.93 \pm 2.49^{\mathrm{b}}$ & $103.10 \pm 19.37^{\mathrm{a}}$ & $16.12 \pm 2.39^{\mathrm{b}}$ & $41.75 \pm 4.84^{\mathrm{ab}}$ & $73.02 \pm 6.32^{\mathrm{a}}$ & $44.73 \pm$ & 30 & 163. \\
\hline 钙 Ca & - & $64.29 \pm$ & $.14 \pm 1$ & $32.09 \pm 0$ & $61.53 \pm 7.40^{\mathrm{b}}$ & $104.40 \pm$ & $24 . / 1$ & (1) & . \\
\hline $\mathrm{Mg}$ & $1 \pm 2.71^{\mathrm{c}}$ & $36.84 \pm 4.30^{\mathrm{b}}$ & $04 \pm 10.79^{a}$ & $27.31 \pm 4.10^{\mathrm{c}}$ & $42.64 \pm 3.70^{b}$ & $63.63 \pm 6.28^{\mathrm{a}}$ & $24.20 \pm 2.34^{\mathrm{c}}$ & $37.55 \pm 2.86^{b}$ & 39.22 \\
\hline 铁 $\mathrm{Fe}$ & $6 \pm 0.20^{\mathrm{b}}$ & $2.64 \pm 0.33^{\mathrm{a}}$ & $3.21 \pm 0.26^{\mathrm{a}}$ & $2.63 \pm 0.21^{\mathrm{b}}$ & $41 \pm 0.28^{\mathrm{a}}$ & $3.43 \pm 0$. & $1.70 \pm 0.20^{\mathrm{c}}$ & $2.77 \pm c$ & $4.33 \pm$ \\
\hline 铜 $\mathrm{Cu}$ & \pm 0 & $2.28 \pm 0.2$ & $8 \pm 0$ & 12 & $50 \pm 0.56^{\mathrm{a}}$ & $61 \pm 0$ & $1.64 \pm 0$ & 2.04 & $2.37+0.0$ \\
\hline Z & \pm 0.03 & 0.08 & $0.26 \pm 0.05^{\mathrm{b}}$ & 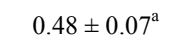 & $\pm 0.07^{b}$ & 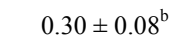 & $4^{a}$ & 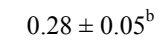 & $3=0.0$ \\
\hline 锰 Mn & $2 \pm 0.23^{\mathrm{b}}$ & $0.86 \pm 0$. & $10 \pm$ & $0.50-0.10$ & $.08^{\mathrm{b}}$ & $5^{\mathrm{a}}$ & $0.35 \pm 0.02^{b}$ & 0.47 & 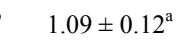 \\
\hline$P$ & $67.26 \pm 4.58^{\mathrm{a}}$ & $35.04 \pm 2.63^{\mathrm{b}}$ & $26.52 \pm 1.06^{\mathrm{C}}$ & $41.09 \pm 5.89^{\mathrm{a}}$ & $22.40 \pm 2.24^{\mathrm{b}}$ & $15.97 \pm 1.85^{\mathrm{c}}$ & $39.47 \pm 3.45^{\mathrm{a}}$ & $30.31 \pm 4.23^{\mathrm{b}}$ & $20.60 \pm 2$. \\
\hline 月 $\mathrm{B}$ & $2.48 \pm 0.68^{\mathrm{a}}$ & $1.49 \pm 0.64^{\mathrm{a}}$ & $1.55 \pm 0.45^{\mathrm{a}}$ & $0.96 \pm 0.60^{\mathrm{a}}$ & $0.95 \pm 0.20^{\mathrm{a}}$ & $0.86 \pm 0.21^{\mathrm{a}}$ & $1.55 \pm 0.31^{\mathrm{a}}$ & $2.42 \pm 0.81^{\mathrm{a}}$ & $2.00 \pm 0.29$ \\
\hline
\end{tabular}

不同小写字母表示不同退化草地间差异显著 $(p<0.05)$ 。

Different lowercase letters indicate significant differences among the different degrees of degraded grasslands $(p<0.05)$.

化趋势相同，都是随叶位的降低呈现增加的趋势 (表2)。与功能叶和老叶相比, $\mathrm{Fe} 、 \mathrm{Cu}$ 含量在幼叶中 含量最低, 但Zn含量最高(表2)。大量的Mn聚集在老 叶中(表2)。P含量随叶位的降低呈现逐渐下降的趋 势，而B含量变化不明显(表2)。

\section{3 讨论}

\section{1 土壤盐碱及芦苇生长特征}

随着全球气候变暖和人类活动, 使得草地退化 严重, 土壤盐碱化加剧, 造成草地生态系统支离破 碎, 生物多样性遭到严重破坏(郑慧莹和李建东, 1995)。本研究结果表明, $\mathrm{Na}^{+}$含量、总盐分含量和 $\mathrm{pH}$ 值是松嫩草地土壤盐碱化的主要影响因子, 本研究
从芦苇生境及盐碱地利用角度, 依据主要影响因子 的差异将松嫩草地分为 3 级, 正常草地、中度退化草 地和严重退化草地, 即随草地退化程度的加剧, 土 壤盐碱化程度越重, 土壤有毒离子 $\mathrm{Na}^{+}$的含量逐级 增加越明显, $\mathrm{Ca}^{2+}$ 含量呈现下降趋势。本研究结果显 示, 3 种生境中芦苇单株高度及生物量性状有较大的 差异性, 其中, 株高和生物量从正常草地到中等退 化草地分别降低了 $44.34 \%$ 和 $48.04 \%$ ，芦苇生长受到 明显抑制, 与之前有关的研究结果 (刘秀香和杨允 菲, 2012；严德福等, 2012)相似。

\section{2 不同生境下芦苇叶片营养元素积累}

高等植物通常将进入体内的大量 $\mathrm{Na}^{+}$积累在成 熟器官中, 从而减少 $\mathrm{Na}^{+}$对新器官的毒害作用, 维 
持自身生理代谢功能，以此来适应盐胁迫环境 (Nakamura et al., 1996; Yasar et al., 2006; 张选等, 2015)。本研究发现随着草地退化程度的加重, 相同 叶位叶片中 $\mathrm{Na}$ 含量呈增加趋势，与此同时，叶位越 低的叶片积累的 $\mathrm{Na}$ 越多(表2)。 $\mathrm{Na}$ 是盐碱土中主要的 致害因子, 芦苇功能叶和老叶具有相对成熟的液泡, 大量 $\mathrm{Na}$ 积累于发达的液泡中以进行渗透调节并保 证胞质代谢活动的正常进行。另外, 幼叶处于生长 阶段, 具有较强的稀释作用, 使得 $\mathrm{Na}$ 含量相对较 低。 $\mathrm{Na}$ 和 $\mathrm{K}$ 具有相似的水合离子半径通道, 在盐胁 迫下它们相互竞争(Munns \& Tester, 2008)。以往有 研究表明, 随盐胁迫强度增加, 芦苇叶片中 $\mathrm{Na}$ 含量 上升，但K含量没有明显的变化(赵可夫等，1998; 宋建等, 2010)。但本研究结果显示, 大量 $\mathrm{Na}$ 流入抑 制了芦苇对 $K$ 的吸收, 这可能是松嫩草地盐碱土具 有高 $\mathrm{pH}$ 特征, 高 $\mathrm{pH}$ 破坏了离子平衡抑制了根系对 $\mathrm{K}$ 的吸收。芦苇成熟叶片在盐碱胁迫下积累大量 $\mathrm{Ca}$, 这有利于缓解 $\mathrm{Na}$ 对幼叶的毒害作用。Ca在盐碱胁迫 下充当第二信使, $\mathrm{Ca}^{2+}$ 含量升高有利于促进 $\mathrm{Na}^{+} / \mathrm{H}^{+}$ 逆向转运蛋白活性, 将大量的 $\mathrm{Na}$ 区隔化在液泡内 (Zhu, 2003; 王芳等, 2012; 张选等, 2015)。Mg、Fe、 $\mathrm{Cu}$ 元素是形成叶绿素所必需的, 尤其是 $\mathrm{Mg}$ 元素作 为叶绿素中为唯一的金属元素, 在植物光合作用中 发挥着重要作用(刘芷宇等, 1982; 王芳等, 2004; 张 进等, 2007; Kobayashi \& Nishizawa, 2012)。本研究 发现, 随叶位的降低芦苇叶片中 $\mathrm{Mg} 、 \mathrm{Fe} 、 \mathrm{Cu}$ 含量升 高, 这可能是由于功能叶片和老叶是进行光合作用 的主要场所, 大量 $\mathrm{Mg} 、 \mathrm{Fe} 、 \mathrm{Cu}$ 促进叶绿素合成并保 证叶片功能, 维持芦苇正常的光合作用, 使植株正 常生长。张玉先(2005)和戴金等(2009)的研究结果表 明, 增施Mn可以提高植物的耐盐性, 本研究结果可 以看出, $\mathrm{Mn}$ 在老叶中大量积累, 这可能是老叶自我 保护机制之一。Zn参与植物体内多种代谢途径, 包 括碳水化合物代谢、蛋白质代谢、植物生长素代谢, $\mathrm{Zn}$ 对植物繁殖器官的形成及细胞膜的功能和结构 有很大影响(刘芷宇等, 1982; 张进等, 2007)。与功能 和老叶片相比较, 芦苇幼叶中积累大量的 $\mathrm{Zn}$, 这有 利于吲哚乙酸等植物生长素的形成, 促进幼叶的生 长(陆景陵, 2003; 潘瑞炽, 2004)。 $\mathrm{P}$ 是植物体内的主 要营养元素, 芦苇叶片 $\mathrm{P}$ 含量随叶位的升高而增加, 幼叶积累的P不仅可以提供生长所需的营养, 同时 还提高了其抗盐碱性, 这可能是芦苇在盐碱胁迫下
的响应策略。

\section{4 结论}

芦苇从土壤中获取生长、发育所必需的矿质元 素, 且矿质元素在各器官中的分布具有明显的选择 性。芦苇具有一定程度的耐盐碱性, 在较高盐碱化 土壤中仍能正常生长繁殖。对芦苇幼叶、功能叶、 老叶的 $\mathrm{K} 、 \mathrm{Na} 、 \mathrm{Ca} 、 \mathrm{Mg} 、 \mathrm{Fe} 、 \mathrm{Cu} 、 \mathrm{Zn} 、 \mathrm{Mn} 、 \mathrm{P} 、 \mathrm{~B}$ 进行分析比较, 结果表明 $\mathrm{Na} 、 \mathrm{Ca}$ 含量随叶位的升高 而呈现降低的趋势, 老叶中含量最高, 这可能是芦 苇对盐碱胁迫的一种适应性响应。功能叶、老叶中 积累大量 $\mathrm{Mg} 、 \mathrm{Fe} 、 \mathrm{Cu}$, 主要是保护叶绿体功能, 使 光合作用正常进行。芦苇在受到盐碱胁迫过程中幼 叶积累更多的 $\mathrm{K}$ 和 $\mathrm{P}$ 来提高光合作用效率, 促进体 内糖类化合物的形成, 加速细胞分裂加快生长, 增 强自身抗逆性和抗病能力。Zn在幼叶中的含量增加, 有利于促进幼叶的生长, 增强幼叶的抗逆性; 而 $\mathrm{Mn}$ 的大量积累可能是老叶的一种自我保护机制。

\section{参考文献}

Asaeda T, Nam LH, Hietz P, Tanaka N, Karunaralne S (2002). Seasonal fluctuations in live and dead biomass of Phragmites australis as described by a growth and decomposition model: Implications of duration of aerobic conditions for litter mineralization and sedimentation. Aquatic Botany, 73, 223-239.

Dai X, Zhang BJ, Zhang Q, Mao SG (2009). Effect of different concentrations of manganese treatments on the growth of wheat seedlings and the activities of SOD·POD under salt stress. Journal of Anhui Agriculture Science, 37, 1300013001. [戴金金, 张边江, 章琦, 毛善国 (2009). 盐胁迫下 不同浓度的锰处理对小麦幼苗生长及 SOD·POD的影响. 安徽农业科学, 37, 13000-13001.]

Hai X, Wan ZJ, Yu SP, Lou XZ, Sun GY (2004). The restoration of wetlands in Songnen Plain 150 years ago. Journal of Northeast Normal University Natural Science Edition, 36(2), 75-81. [海霞, 万忠娟, 于少鹏, 罗新正, 孙广友 (2004). 松嫩平原距今 150 年湿地景观结构重建. 东北师 大学报自然科学版, 36(2), 75-81.]

Kiviat E (2013). Ecosystem services of phragmites in North America with emphasis on habitat functions. Annals of Botany Plants, 5, 1-29.

Kobayashi T, Nishizawa NK (2012). Iron uptake, translocation, and regulation in higher plants. Annual Review of Plant Biology, 63, 131-152.

Li XJ, Li QS, Wang ZC, Liu XT (2002). A research on characteristics and rational exploitation of soda saline land in the 
western Songnen Plain. Research of Agricultural Modernization, 23, 361-400. [李秀军, 李取生, 王志春, 刘兴 土 (2002). 松嫩平原西部盐碱地特点及合理利用研究. 农业现代化研究, 23, 361-400.]

Liu XX, Yang YF (2012). Allometry analysis of reproductive ramets of Phragmites australis populations from different habitats in the Songnen Plain of China. Acta Prataculturae Sinica, 21, 313-318. [刘秀香, 杨允菲 (2012). 松嫩平原 不同生境芦苇生殖分株的异速生长分析. 草业学报, 21, 313-318.]

Liu ZY, Tang YL, Luo ZC (1982). Atlas of Major Crop Malnutrition. Agriculture Press, Beijing. 47-48. [刘芷宇, 唐 永良, 罗质超 (1982). 主要作物营养失调症状图谱. 农 业出版社, 北京. 47-48.]

Lu JL (2003). Plant Nutriology. China Agricultural University Press, Beijing. [陆景陵 (2003). 植物营养学. 中国农业 大学出版社, 北京.]

Munns R, Tester M (2008). Mechanisms of salinity tolerance. Annual Review of Plant Biology, 59, 651-681.

Nakamura T, Ishitani M, Harinasut P, Nomura M, Takabe T, Takabe T (1996). Distribution of glycinebetaine in old and young leaf blades of salt-stressed barley plants. Plant and Cell Physiology, 37, 873-877.

Pan RC (2004). Plant Physiology. Higher Education Press, Beijing. [潘瑞炽 (2004). 植物生理学. 高等教育出版 社, 北京.]

Qiu T (2014). Biological and ecological characterization of Phragmites australis in Songnen Prairie. Acta Pratacultural Science, 31, 300-305. [邱天 (2014). 松嫩平原芦苇 的生物学和生态学特征. 草业科学, 31, 300-305.]

Song J, Yang YX, Nie LL, Zhang Y, Liu ZQ (2010). Comparative study on physiological characteristics of salt tolerance between Phragmites communis and Puccinellia tenuiflora. Tianjin Agricultural Sciences, 16(6), 10-12. [宋建, 杨迎 霞, 聂莉莉, 张越, 刘仲齐 (2010). 芦苇和碱茅耐盐生 理特性的比较分析. 天津农业科学, 16(6), 10-12.]

Wang F, Liu P, Zhu JW (2004). Effect of magnesium (Mg) on root activity, chlorophyll content and membrane permeability of soybean. Journal of Agro-Environment Science, 23, 235-239. [王芳, 刘鹏, 朱靖文 (2004). 镁对大豆根 系活力叶绿素含量和膜透性的影响. 农业环境科学学 报, 23, 235-239.]

Wang F, Wan SB, Meng QW, Li XG (2012). Regulation of $\mathrm{Ca}^{2+}$ in plant response mechanisms under salt stress. Life Science Research, 16, 362-367. [王芳, 万书波, 孟庆伟, 李新国 (2012). $\mathrm{Ca}^{2+}$ 在植物盐胁迫响应机制中的调控作 用. 生命科学研究, 16, 362-367.]

Xia J, Wishart DS (2011). Web-based inference of biological patterns, functions and pathways from metabolomic data using MetaboAnalyst. Nature Protocols, 6, 743-760.

Xiang M, Ye HC, Li GF, Chen JL (2000). Comparison of some characteristics between Phragmites communis and its salt tolerant variant. Acta Botanica Sinica, 42, 1126-1130. [祥 明, 叶和春, 李国凤, 陈建林 (2000). 芦苇耐盐变异体 与野生型植株某些特性的比较. 植物学报, 42, 11261130.]

Yan DF, Yang YF, Zhao MQ (2012). Phenotypic plasticity of leaves on reed populations in heterogeneous habitats in the Songnen Plains of China. Journal of Northeast Normal University (Natural Science Edition), 44(2), 78-83. [严德 福, 杨允菲, 赵明清 (2012). 松嫩平原异质生境芦苇种 群叶片的表型可塑性. 东北师大学报(自然科学版), 44(2), 78-83.]

Yang F, Deng W, Yang JF, Zhang GX, Li XJ (2006). Effects of soil moisture and electrical conductivity on growth and population distribution of Phragmites australis. Journal of Soil and Water Conservation, 20, 199-201. [杨帆, 邓伟, 杨建锋, 章光新, 李秀军 (2006). 土壤含水量和电导率 对芦苇生长和种群分布的影响. 水土保持学报, 20 , 199-201]

Yang YF, Lang HQ (1998). A study of population regulation of Phragmites communis as a clonal plant in different ecological conditions. Acta Pratacultural Science, 7(2), 1-9. [杨允菲, 郎惠卿 (1998). 不同生态条件下芦苇无性系 种群调节分析. 草业学报, 7(2), 1-9.]

Yasar F, Uzal O, Tufenkci S, Yildiz K (2006). Ion accumulation in different organs of green bean genotypes grown under salt stress. Plant Soil and Environment, 52, 476480.

Zhang J, Wu LH, Wang MY (2007). Effect of iron and nitrogen mixed fertilizers on the content of $\mathrm{Fe}, \mathrm{Zn}, \mathrm{Ca}, \mathrm{Mg}$ and protein in brown rice. Journal of Agro-Environment Science, 26, 122-125. [张进, 吴良欢, 王敏艳 (2007). 铁氮 配施对稻米中铁、锌、钲、镁和蛋白质含量的影响. 农 业环境科学学报, 26, 122-125.]

Zhang SG, Qiu JJ, Zhang H (2000). Salinized soil resources in China and their comprehensive harnessing. Chinese Journal of Agricultural Resources and Regional Planning, 21(1), 52-56. [张士功, 邱建军, 张华 (2000). 我国盐渍 土资源及其综合治理. 中国农业资源与区划, 21(1), 52-56.]

Zhang X, Xiao XL, Li PB, Sun YF, Guo WY, Yao YA (2015). Physiological responses of Populus alba var. pyramidalis leaves with different maturity to alkali stress. Scientia Silvae Sinicae, 51(12), 9-16. [张选, 肖薪龙, 李鹏勃, 孙玉 芳, 郭琬䒯, 姚银安 (2015). 新疆杨不同成熟度叶片在 碱胁迫下的生理响应. 林业科学, 51(12), 9-16.]

Zhang YX (2005). Effect of manganese on yield in different soybean genetic types. Chinese Agricultural Science Bulletin, 2, 245-247. [张玉先 (2005). 锰元素对不同基因型 大豆产量的影响. 中国农学通报, 2, 245-247.]

Zhao KF, Feng LT, Zhang SQ (1998). Adaptive physiology of different ecotypes of Phragmites communis to salinity in the Yellow River Delta I. Osmotica and their contributions 
to the osmotic adjustment. Acta Ecologica Sinica, 18, 1-10. [赵可夫, 冯立田, 张圣强 (1998). 黄河三角洲不 同生态型芦苇对盐度适应生理的研究I. 渗透调节物质 及其贡献. 生态学报, 18, 1-10.]

Zhao LP, Shang QC, Li CL (2000). Present situation and problems of sodic soil improvement and using in Songliao Plain. Journal of Jilin Agricultural University, 22(Suppl.), 79-83, 85. [赵兰坡, 尚庆昌, 李春林 (2000). 松辽平原 苏打盐碱土改良利用研究现状及问题. 吉林农业大学 学报, 22(Suppl.), 79-83, 85.]

Zheng HY, Li JD (1995). A preliminary study on the formation of saline-alkali plant communities in the Songnen Plain. Acta Phytoecologica Sinica, 19, 1-12. [郑慧荣, 李建东
(1995). 松嫩平原盐碱植物群落形成过程的探讨. 植物 生态学报, 19, 1-12.]

Zhu JK (2003). Regulation of ion homeostasis under salt stress. Current Opinion in Plant Biology, 6, 441-445.

Zhuang Y, Sun YX, Wang ZS, Yang LL, Deng ZF, Yao ZG, An SQ (2010). Research advances in ecotypes of Phragmites australis. Acta Ecologica Sinica, 30, 2173-2181. [庄 瑶, 孙一香, 王中生, 杨琳璐, 邓自发, 姚志刚, 安树青 (2010). 芦苇生态型研究进展. 生态学报, 30, 21732181.]

责任编委: 王锁民 责任编辑: 李 敏 